\title{
3D Numerical investigation of pressure field of an orifice compensated hydrostatic bearing
}

\author{
Faiza Ghezali ${ }^{1, a}$, A. Bouzidane ${ }^{2}$ and M. Thomas ${ }^{3}$ \\ 1 Department of Mechanical Engineering, University of Sidi Bel Abbes, BP 89, Cité Ben M'hidi, Sidi Bel Abbes, Algeria \\ 2 Research Laboratory of Industrial Technologies, Department of Mechanical Engineering, University Tiaret, \\ BP 78 City/Province, Tiaret 14000, Algeria \\ 3 Department of Mechanical Engineering, École de Technologie Supérieure, 1100 Notre-Dame Street West, Montreal, \\ Quebec, (H3C 1K3), Canada
}

Received 22 March 2015, Accepted 25 January 2016

\begin{abstract}
In recent years, much attention has been given to hydrostatic/hybrid journal bearings in research due to their wide spread engineering applications, such as: high speed turbomachinery, machine tools spindles, cryogenic equipment, and precision grinding. However, the activity toward improved understanding of the flow regimes and the associated pressure pattern in the recess flow phenomena is still needed. The objective of this work is to provide an understanding of the influence of regime flow in recess on the pressure field of hydrostatic bearing flat pad fed by orifice restrictor and orifice inertia. 3D Reynolds averaged Navier Stokes equations with the SST-k $\omega$ turbulence model are applied in order to investigate the effects of pressure supply, dynamic viscosity and recess depths on the pressure profile. The finite volume method implemented in the ANSYS-CFX software is used. To prove the robustness of CFD code, a comparison of the numerical results and the Reynolds equation is performed at very deep recess. The results exhibit very good agreements between CFD and Reynolds equation methods. On the other hand, the several cases treated in this work contribute also to analyze and to explain the main reasons providing the inertia and the Rayleigh effect in recess flow.
\end{abstract}

Key words: Pressure field / ANSYS-CFX / 3D- Reynolds averaged Navier Stokes / SST k- $\omega$ / Reynolds equation

\section{Introduction}

The comprehension of flow behavior inside the hydrostatic recess is a subject of several researches. Most of the recent studies are based on the classical Reynolds equation, or its modified forms, where turbulence or variable properties of flow are taken into account. The main operational parameters considered in the several numerical and experimental researches were shaft speed, inlet jet pressure supply and the Reynolds number [1-9]. Furthermore, numerous investigations indicated the significant effects of variations in the geometric structures of hybrid journal bearing systems on performance characteristics [10-16]. Recently, the activity toward improved modeling of the flow in the hydrostatic pocket has increased by using formulation of the 3DNavier Stocks equations [17-20] to provide a better understanding of the physics of flow in the recess.

The objectives of this present paper are to study the influences of regime flow and inertia effect in the recess

\footnotetext{
${ }^{a}$ Corresponding author: faiza_ghezali@yahoo.fr
}

bearing. The first ones consist in dynamic viscosity and inlet jet supply pressure of lubricant. The second one consists in recess depth. The numerical simulations are performed by using 3D Naviers Stockes equations with SST-K $\omega$ model. The results are discussed, analyzed and compared to the Reynolds equation approach at very deep recess. The results are in good agreements with the Reynolds equation method.

\section{Hydrostatic bearing description}

The typical geometry of a square recess of a hydrostatic bearing is shown in Figure 1. This configuration is outfitted at the bottom with the orifice feedline of length $L$ equal to $6 \mathrm{~mm}$. The diameter of a cylindrical orifice through which oil was fed into the hydrostatic recess was set at $D=2.49 \times 10^{-3} \mathrm{~m}$. The main physical parameters that differentiate the recess is their recess depth over film thickness $e / h_{0}\left(e=\right.$ recess depth and $h_{0}=$ film thickness $)$ which were varied from $4,8,32$ and 152 . 


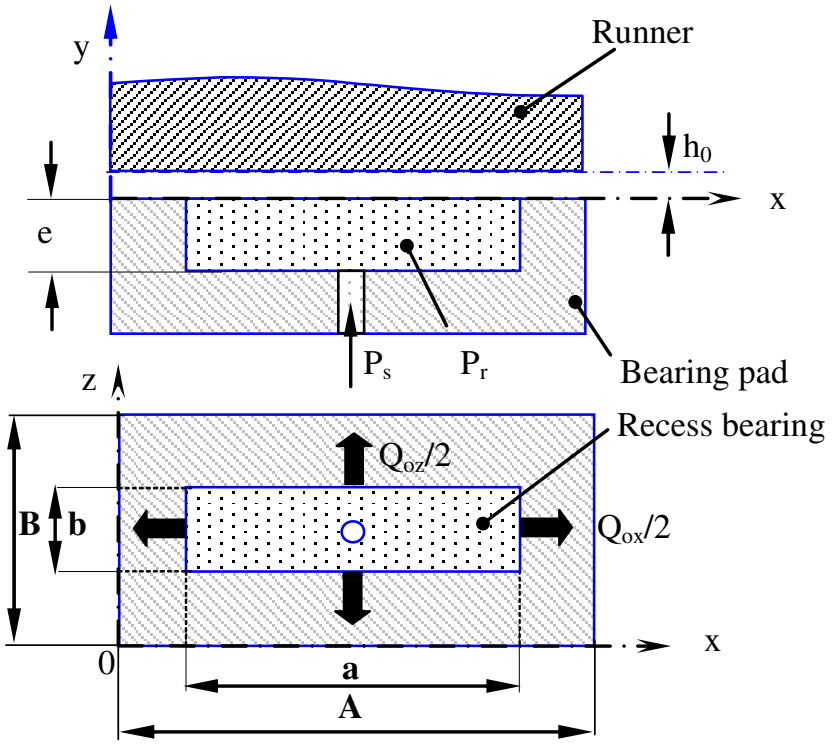

Fig. 1. Rectangular hydrostatic bearing flat pad.

\section{Numerical modeling}

\subsection{Reynolds equation approach}

The Reynolds equation allows for the computation of the pressure distribution $P(x, z, t)$. For an incompressible, laminar, isoviscous, and inertialess fluid, the Reynolds equation may be written as [3]:

$$
\frac{\partial}{\partial x}\left(\frac{\partial P(x, z, t)}{\partial x}\right)+\frac{\partial}{\partial z}\left(\frac{\partial P(x, z, t)}{\partial z}\right)=12 \frac{\mu}{h^{3}} \dot{h}
$$

where: $0 \leq x \leq A$ and $0 \leq z \leq B ; P(x, z, t)$ is the hydrostatic pressure field of the hydrostatic bearing pad; $h$ is the film thickness of the hydrostatic bearing pad; $\dot{h}$ is the squeeze velocity of the hydrostatic bearing pad.

\subsubsection{Reynolds boundary conditions}

In order to solve the Reynolds equation (Eq. (1)), it is assumed that: (i) the recess depth is considered very deep $\left(h_{p}=152 h\right)$; (ii) at the external boundary, nodal pressures are zero; (iii) the nodal pressures for node on the recess are constant and equal to $P_{i}$; (iv) flow of lubricant through the restrictor is equal to the journal bearing input flow; (v) negative pressure is set to zero during the interactive process to take care of oil film cavitations.

\subsubsection{Recess pressure}

The recess pressure, for each hydrostatic bearing pads (Fig. 1), is determined by solving of the following flow continuity equation for pure hydrostatic (no moving surface, $\dot{h}=d h / d t=0)$ :

$$
Q_{\mathrm{r}}=Q_{\mathrm{ox}}+Q_{\mathrm{oz}}
$$

where:

$$
\begin{aligned}
Q_{\mathrm{r}} & =\frac{\pi C_{\mathrm{d}} d_{o}^{2}}{\sqrt{8 \rho}} \sqrt{\left(P_{\mathrm{s}}-P_{\mathrm{r}}\right)} \\
Q_{\mathrm{o} x} & =2 \int_{0}^{B / 2} \int_{0}^{h} u_{x} \mathrm{~d} z \mathrm{~d} y ; Q_{o z}=2 \int_{0}^{A / 2} \int_{0}^{h} u_{z} \mathrm{~d} x \mathrm{~d} y \\
u_{x} & =\frac{1}{2 \mu} \frac{\partial P}{\partial x}(y-h) y ; u_{z}=\frac{1}{2 \mu} \frac{\partial P}{\partial z}(y-h) y
\end{aligned}
$$

where: $d_{\mathrm{o}}$ is the orifice diameter; $\rho$ is the density; $Q_{\mathrm{ox}}$ and $Q_{\mathrm{oz}}$ are the oil flow of the hydrostatic bearing pad in the $x$, and $z$ directions, respectively, $Q_{\mathrm{r}}$ represents the flow through a orifice restrictor; $u_{x}, u_{y}$ and $u_{z}$ are the flow velocities in the $x, y$ and $z$ directions, respectively; $C_{\mathrm{d}}$ is the orifice discharge coefficient. Note that the discharge coefficient $C_{\mathrm{d}}$ is a function of the Reynolds number, Re. Typically it varies in a nonlinear fashion from $C_{\mathrm{d}} \approx 0.3$ for $R e \approx 2$ to $C_{\mathrm{d}} \approx 0.7$ at $R e \approx 100$, and drops to about $C_{\mathrm{d}} \approx 0.6$ for higher $R e$ values [20].

It must be noticed that when the orifice length $l$ is no longer negligible, the discharge coefficient $C_{\mathrm{d}}$, can be determined by means of the following equations [20].

$$
\begin{aligned}
& C_{\mathrm{d}}=\left[1.5+13.74\left(\frac{l}{d R e}\right)^{1 / 2}\right]^{-1 / 2} \text { for } \frac{d R e}{l}>50 \\
& C_{\mathrm{d}}=\left[2.5+64\left(\frac{l}{d R e}\right)\right]^{-1 / 2} \text { for } \frac{d R e}{l}<50
\end{aligned}
$$

The feeding Reynolds number for an orifice is as follows [21]:

$$
R e=\frac{d_{0}}{\mu}[2 \rho(P s-P r)]^{1 / 2}
$$

\subsection{Computational fluid dynamics}

Over the past decade, computational fluid dynamics (CFD) has become the preferred modeling technique for fluid dynamics analysis. CFD can provide a good understanding of the flow characteristics in hydrostatic journal bearings. In this study, commercial CFD software (Ansys CFX 12.0.1) is used to provide the numerical solution.

In this present model, the cavitation is not considered since the minimum pressure value never drops above the vapor pressure. The conservation equation for steady state incompressible, isoviscous and isothermal flow, with zero gravitational and other external body forces, are:

- Mass conservation equation

$$
\nabla \cdot v=0
$$

- Momentum equations

$$
\rho(v \cdot \nabla) v=-\nabla p+\nabla \cdot(\mu \nabla v)
$$

where $v=(u, v, w)^{T}$ is the velocity vector, $p$ is the static pressure, $\mu$ is dynamic viscosity, $\rho$ is the density. 


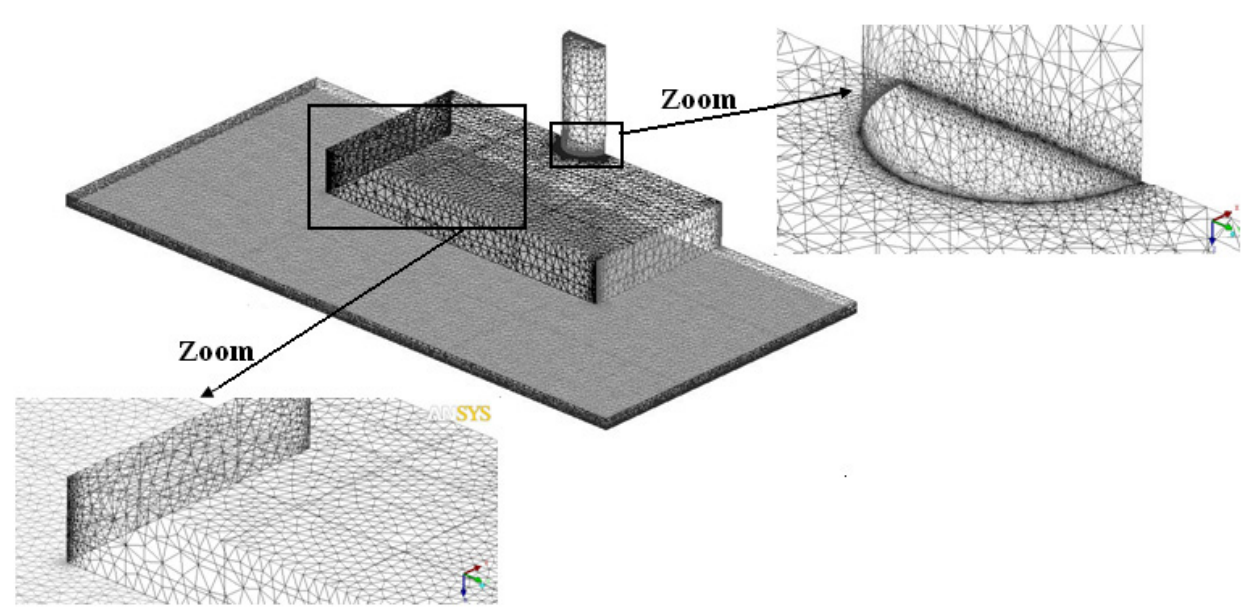

Fig. 2. Perspective views of the computational domain done with the ICEM.CFD.

The 3-D full NSEs (Momentum equations (Eq. (10))) and continuity equations (Mass conservation equation (Eq. (9))) are used to solve the pressure distribution for the hydrostatic bearing. The commercial program used, in this simulation, is the CFD-ANSYS-CFX 12.

\subsubsection{Mesh type and solution procedure}

Numerical simulations on unstructured grids have been performed using a steady state approach with the commercial code ANSYS CFX12.0.1. The closure of the Reynolds averaged equations (RANS) is provided by the shear stress transport (SST-K $\omega$ ). The choice of this model was justified since the model gives highly accurate predictions of the onset and the amount of flow separation under adverse pressure gradients [8]. The continuity and momentum equations are solved on the present configuration by the finite volume method. A close view of the computational unstructured grid from $e / h=4$ is presented in Figure 2. The geometry was gridded by the ICEM-CFD mesh generator included with the CFX-12 software. The grid is concentrated at the outlet of the orifice and near the recess outlet to the start of thin film flow to resolve the pressure pattern in this region.

The computational domain is composed by orifice feed-line, the recess, the sill and the film thickness as shown in Figure 3.

It also may be noticed that all geometrical parameters and the boundary conditions have been reproduced according to the numerical study based on Reynolds equation methods presented in Section 3.1.2 (Reynolds boundary conditions). Regarding the boundary conditions, adiabatic and no slip conditions are used at the wall. The boundary condition wall accumulates the orifice feed-line, the sill and the recess. At the entrance of the orifice, the constant static pressure supply applied and laminar regime flow was imposed. At the outlet, the reference pressure is set to absolute zero (Pascal). Due to the flow symmetry at the axial ends of the model, only one half of the

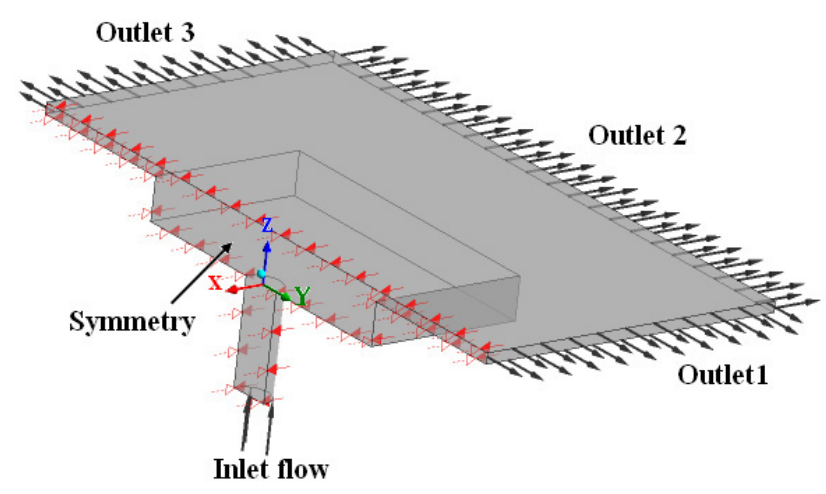

Fig. 3. Computational domain and boundary conditions.

geometry in the axial direction is considered and a symmetry boundary condition is applied. Convergence was attained when the RMS (root-mean-squared) residual values of all flow parameters $\left(u, v, w\right.$ and $p$ ) fell below $10^{-5}$. The total numbers of elements and nodes used for this configuration are 882375 and 161804 respectively. This grid was chosen after several tests of the studies cases. It noted that other denser grids were used for the other geometric parameters $(e / h)$.

\section{Numerical results}

3D Reynolds averaged Navier Stokes equations with the SST-k $\omega$ turbulence model were applied to investigate the effects of pressure supply, dynamic viscosity and recess depths on the pressure profile of hydrostatic bearing flat pad in both laminar and no-laminar regime. The finite volume method implemented in the ANSYS-CFX software is used. To prove the robustness of CFD code, a comparison of the numerical results and the Reynolds equation, which is restricted to only laminar regime, is performed at very deep recess. The several cases treated in this work contribute also to analyze and to explain the main reasons for providing the inertia and the Rayleigh 

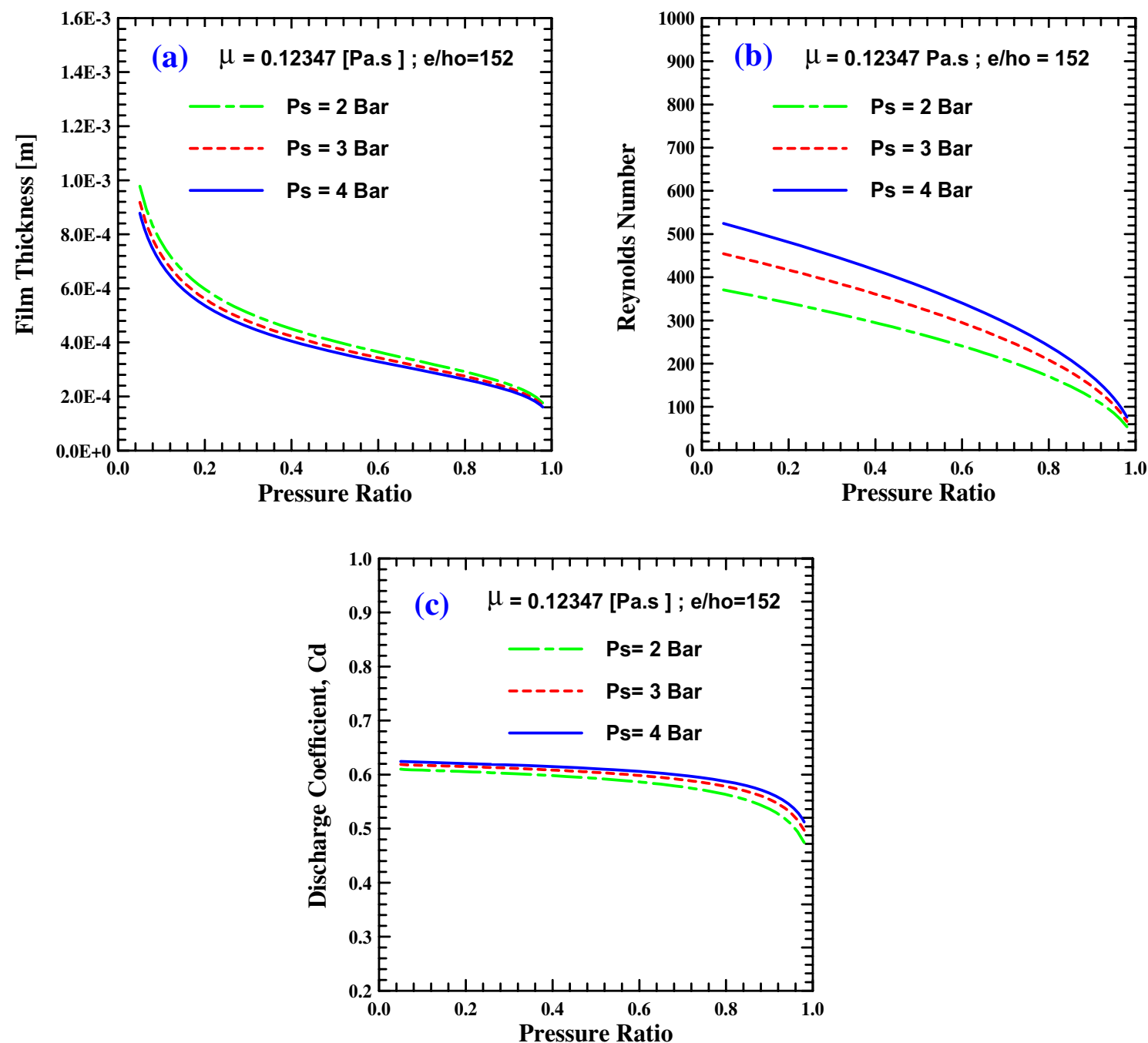

Fig. 4. Film thickness, feeding Reynolds number and recess pressure versus pressure ratio for different values of supply pressure predicted from Reynolds equation.

effect in recess flow. Two numerical investigations are developed in this paper. The first one is based on resolution of Reynolds equation and the second one resolved the three dimensional Naviers Stockes equations integrated on Ansys-CFX-12.0 software. The bearing characteristics are the following: bearing pad length $A$ is $0.037 \mathrm{~m}$, bearing pad width $B$ is $0.037 \mathrm{~m}$, dimension ratio $a / A=b / B$ is 0.5 ; restrictor diameter $d_{\mathrm{o}}$ is $2.49 \mathrm{~mm}$ and restrictor length $l$ is $6 \mathrm{~mm}$.

\subsection{Effect of the pressure supply and the viscosity on the film thickness, recess pressure and feeding Reynolds number predicted from Reynolds approach}

The evolutions of film thickness, Reynolds number and discharge coefficient are studied according to the pressure ratio for different pressure supply and two dynamic viscosities in hydrostatic bearing flat pad. These results were computed using the Reynolds approach at very deep recess $(e / h=152)$. Figures $4 \mathrm{a}-4 \mathrm{c}$ illustrate the effects of the pressure ratio and supply pressure on the film thickness, Reynolds number and discharge coefficient for a viscosity of $0.12347 \mathrm{~Pa}$.s. These figures show that the increase of supply pressure from 2 to 4 Bar decreases the film thickness and increases the Reynolds number and discharge coefficient. It must be noticed that when the film thickness is fixed, the pressure ratio decreases with increases in supply pressure. On the other hand, as seen in Figures $4 \mathrm{a}-4 \mathrm{c}$, the increase in the pressure ratio decreases the film thickness, Reynolds number and discharge coefficient. Further, with an increase of pressure supply, the Reynolds number and discharge coefficient increase slightly. This effect is usually offset by purposefully providing more flow by increasing of supply pressure.

The influence of the viscosity and pressure ratio on the film thickness, Reynolds number and discharge coefficient, for a supply pressure set to 2 Bar, are presented in Figures $5 \mathrm{a}-5 \mathrm{c}$. These curves show that the increase of viscosity increases the film thickness and decreases the Reynolds number and discharge coefficient. It was 

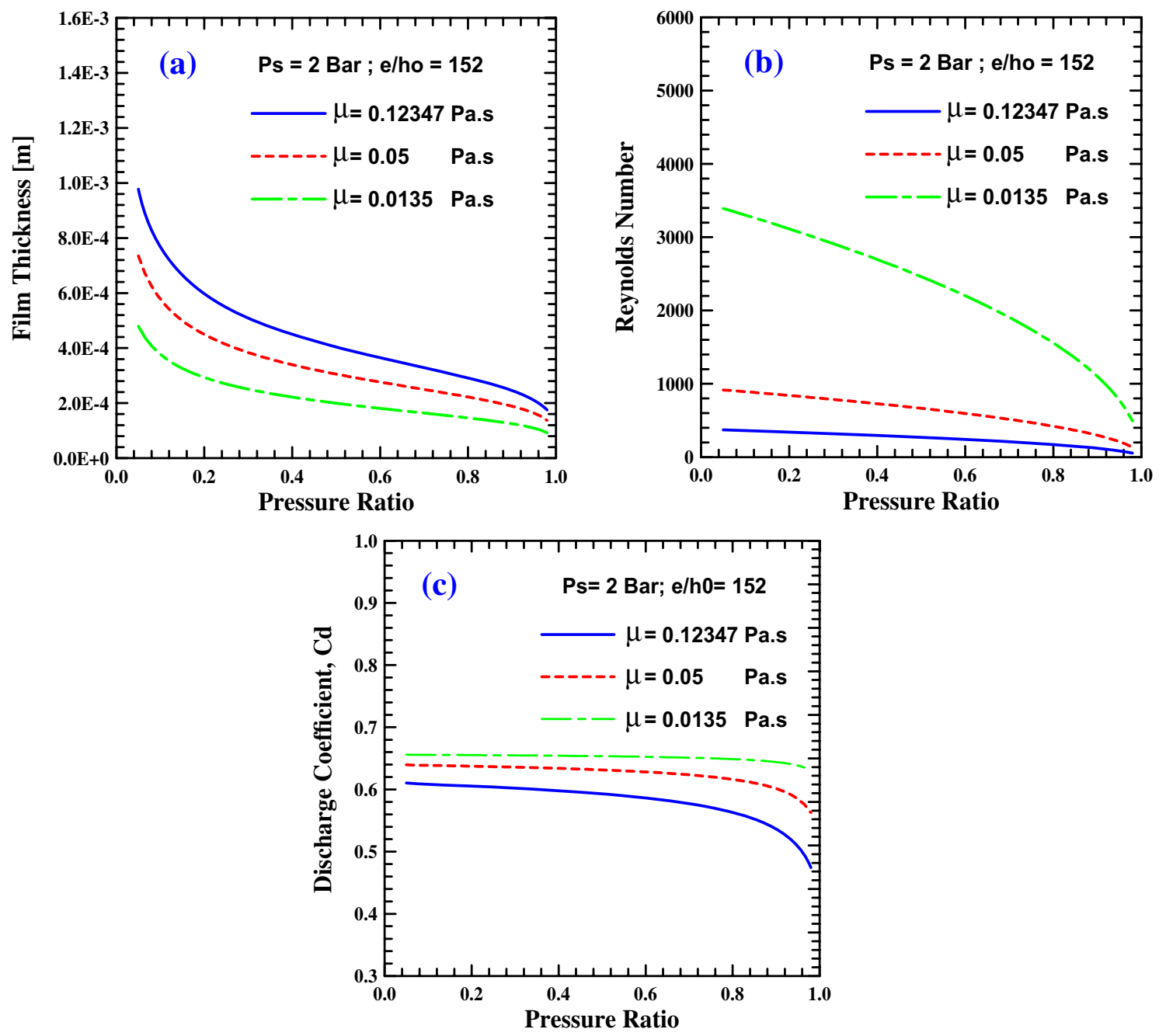

Fig. 5. Film thickness, feeding Reynolds number and discharge coefficient versus pressure ratio for different viscosity predicted from Reynolds equation.

observed that when the film thickness is fixed, the pressure ratio increases with an increase in viscosity. In this case, it must be noticed that the Reynolds number and discharge coefficient decreases significantly due to the increase of viscosity.

\subsection{Effect of the pressure supply, the viscosity and deep recesses on static pressure predicted from CFD}

\subsubsection{Comparison between Reynolds equation and CFD-ANSYS-CFX 12.0 models}

To check the validity of the numerical analysis, the numerical results predicted from CFD-CFD-ANSYS-CFX 12.0 model were computed and compared with those obtained by the Reynolds equation model which is restricted to only laminar flow in recess of a hydrostatic bearing.

Figure 6a shows a comparison between Reynolds equation and CFD-CFD-ANSYS-CFX 12.0 results at the middle of the hydrostatic bearing. The results show good agreements between CFD and Reynolds equation methods. Figure $6 \mathrm{~b}$ describes the variation of pressure distribution from Reynolds equation for a bearing pad dimension ratio of $A / B=1$, recess dimension ratio of $a / A=b / B=0.5$, supply pressure of 2 Bar and a film thickness of $0.337 \mathrm{~mm}$ at very deep recess $(e / h=152)$. These curves show that the pressure distribution is spread along the entire bearing pad area, without concentration of pressure. As seen in Figure $6 \mathrm{~b}$ that the pressure is almost constant in recess bearing, then it is subject to a very rapid pressure decrease and after a development length located at the beginning of the thin film, the pressure decrease as a linear variation at the middle of the hydrostatic bearing as shown in Figure 6c. However at the edges, the pressures are curved.

\subsubsection{Effect of the supply pressure on static pressure predicted from CFD}

Figures $7 \mathrm{a}$ and $7 \mathrm{~b}$ illustrate the effect of the supply pressure on the static pressure predicted from 


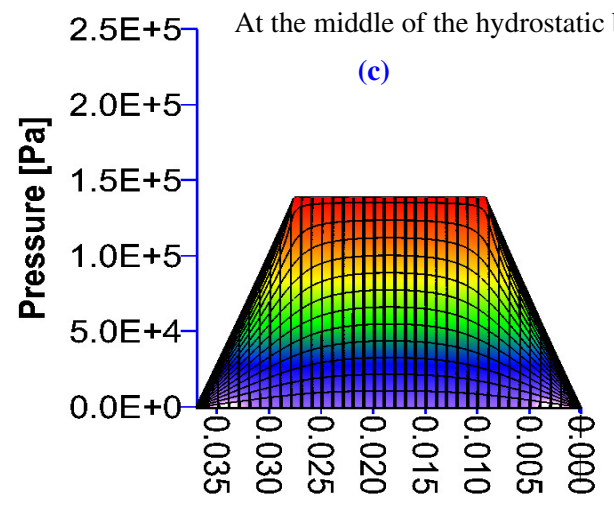

A $[\mathrm{m}]$
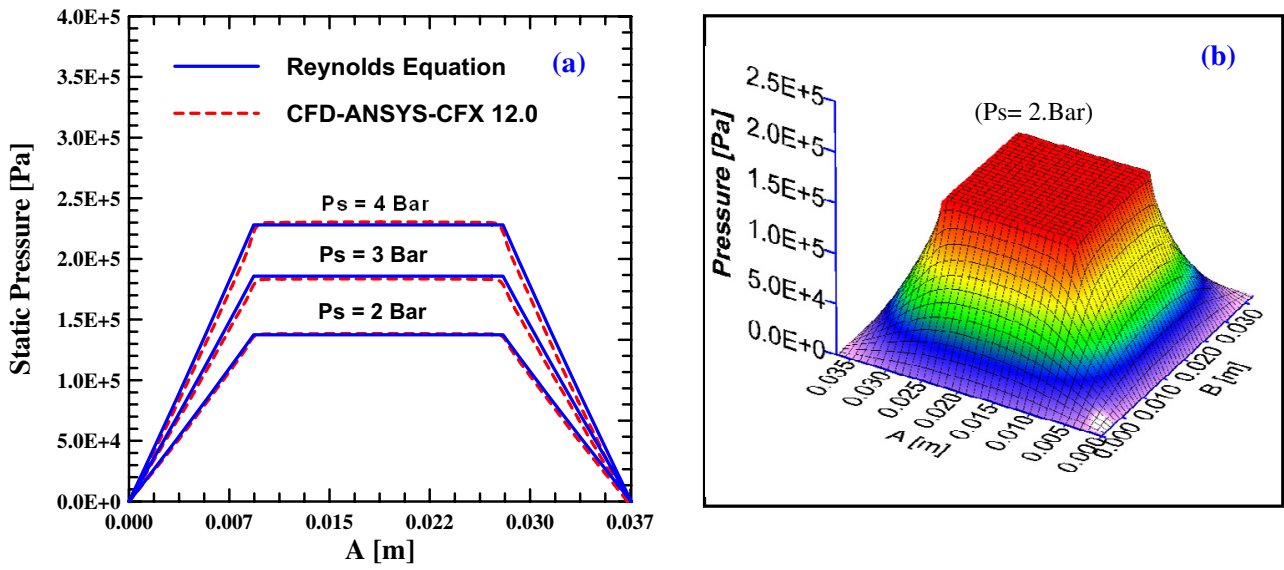

Fig. 6. Pressure profile $\left(h_{0}=0.337 \mathrm{~mm}\left(\beta_{0}=0.69\right)\right.$ : (a) comparison of pressure profile at the axial centreline; (b) pressure distribution predicted from Reynolds equation.
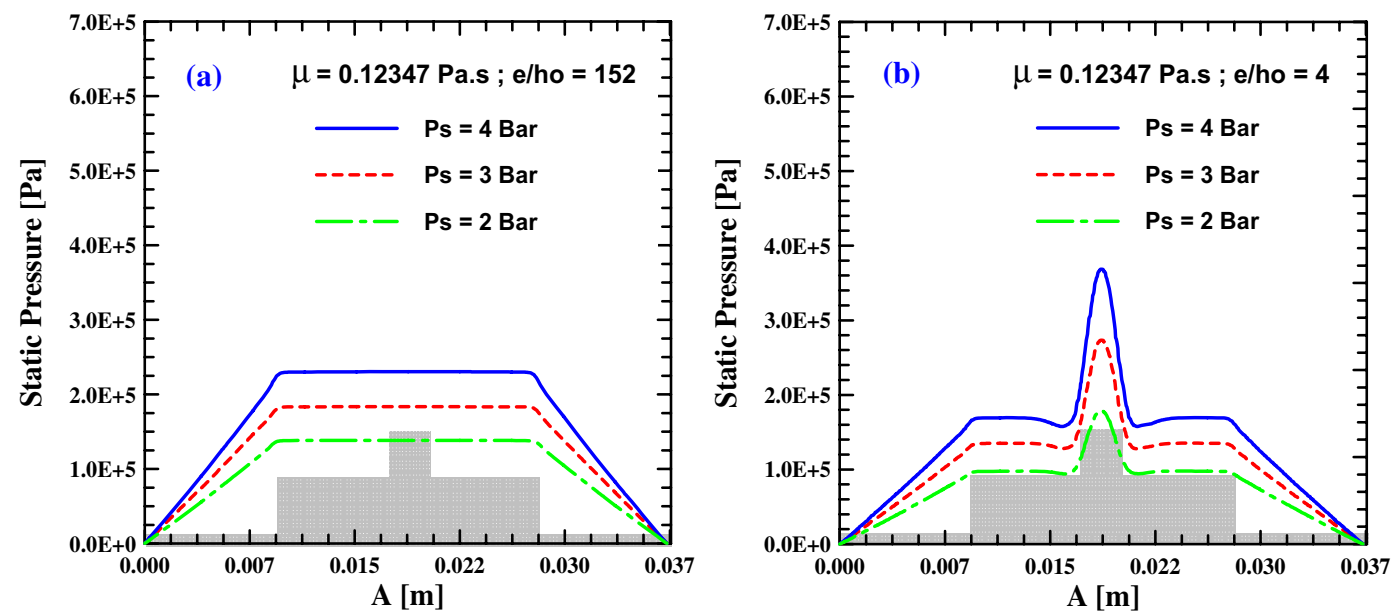

Fig. 7. Influence of the supply pressure on static pressure predicted from CFD- ANSYS-CFX 12.0 .

CFD-ANSYS-CFX 12.0., for a viscosity of $\mu=$ 0.12347 Pa.s. and a film thickness of $0.337 \mathrm{~mm}$.

Figure $7 \mathrm{a}$ presents the influence of supply pressure on static pressure for deep recessed bearing depth of $e / h_{0}=152$. These results demonstrate that the increase of the supply pressure from 2 to 4 Bar increases the static pressure due to the increase in recess pressure. These results are confirmed by Reynolds equation as seen in
Figure 4c. It should be noticed that the recess pressure distribution tends to become constant, which signifies that the jet flow is completely diffused in the recess bearing. The influence of supply pressure on static pressure for shallow recessed bearing of $e / h_{0}=4$ is presented in Figure $7 \mathrm{~b}$. These results reveal that the increase of the supply pressure, from 2 to 4 Bar, results in higher concentrated pressure peaks close to the restrictor area due 

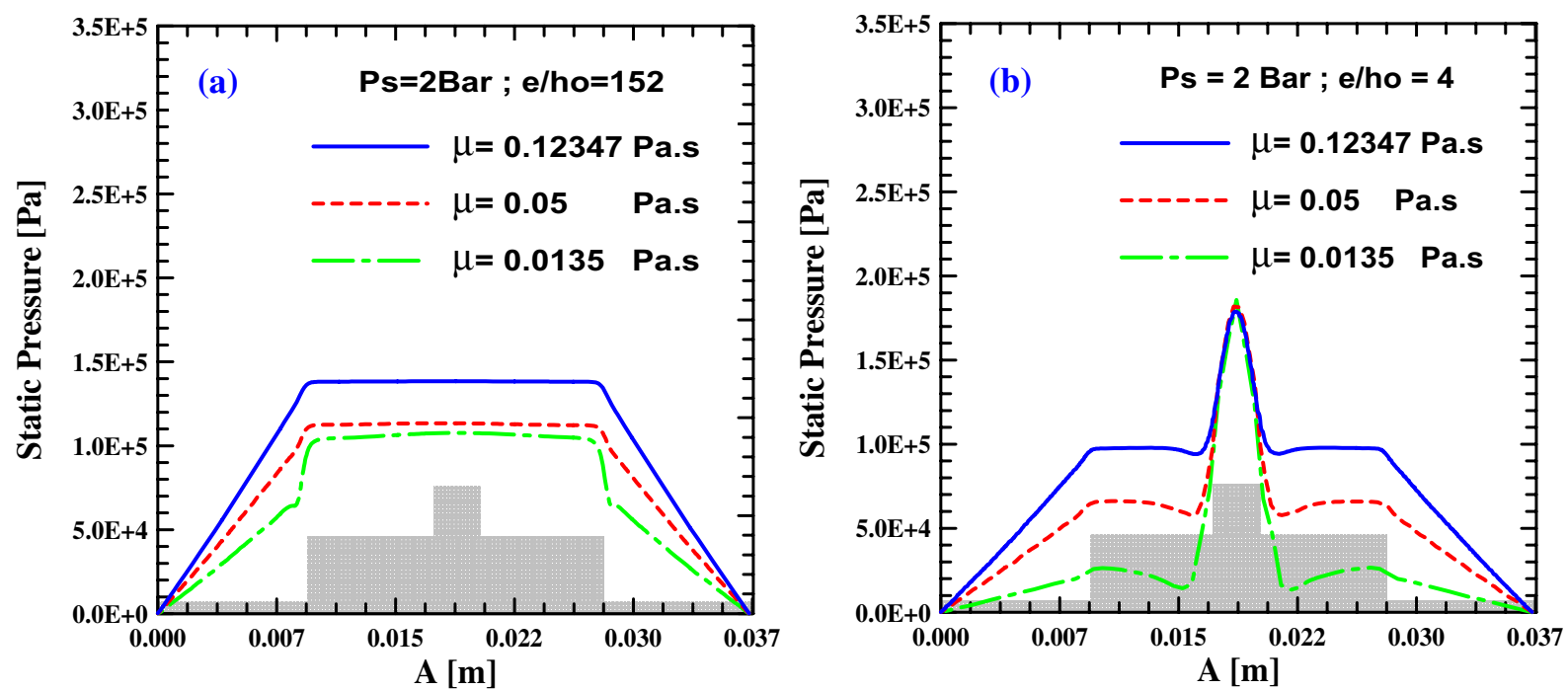

Fig. 8. Influence of the viscosity on static pressure predicted from CFD- ANSYS-CFX 12.0.

to the high feeding Reynolds number which increases with an increase in supply pressure (Fig. 4b).

\subsubsection{Effect of the viscosity on static pressure predicted from CFD}

Figures $8 \mathrm{a}$ and $8 \mathrm{~b}$ presents the effect of the viscosity on the static pressure predicted from CFD-ANSYS-CFX 12.0., for a supply pressure of 2 Bar and two recess depth: (a) $e / h_{0}=4$ and (b) $e / h_{0}=152$.

These results reveal that the pressure between the restrictor area (injection zone) and the end of the recess, for shallow recessed bearing $\left(e / h_{0}=4\right)$ decreases comparing to those obtained in deep recessed bearing $\left(e / h_{0}=152\right)$. However, the pressure close to the restrictor area, for shallow recessed bearing $\left(e / h_{0}=4\right)$ increases comparing to those obtained in deep recessed bearing $\left(e / h_{0}=152\right)$. Figure 8a shows the influence of viscosity on static pressure for deep recessed bearing of $e / h_{0}=152$. For this case, the results obtained from CFD demonstrate that the increase of the viscosity, from 0.0135 to 0.12347 Pa.s, increases the static pressure due to the increase in both recess pressure and feeding Reynolds number, because the film thickness is considered constant as confirmed in results obtained from Reynolds approach (Fig. 5). A pressure drop is observed close to the step recess bearing presents a concentrated inertia effects when the viscosity decreases. This phenomenon can be explained by high variations of pressure when passing from the recess to the film thickness. The influence of viscosity on static pressure, for shallow recessed bearing depth of $e / h_{0}=4$, is presented in Figure 8b. These results demonstrate that the increase of the viscosity, from 0.0135 to 0.12347 Pa.s, results in higher concentrated pressure peaks close to the restrictor area. This phenomenon can be explained by increasing of feeding Reynolds number which resulted from a decrease in viscosity (Fig. 5b). On the other hand, a pressure loss is observed, between the restrictor area (injection zone) and the end of the recess, when the viscosity decreases. This pressure loss is due to the Rayleigh step effect resulted to the large developed Main Vortical Cell (MVC). These results were confirmed by Horvat and Frêne $[19,20]$. It must be noticed that the pressure in the recess area is not uniform.

\subsubsection{Effect of the deep recess on static pressure predicted from CFD}

Figures $9 \mathrm{a}$ and $9 \mathrm{~b}$ presents the distribution of the static pressure obtained from CFD-ANSYS-CFX 12.0 for different deep recess and a supply pressure of 2 Bar. These results serve to compare the influence of recess depth in pressure fields using two values of dynamic viscosities $\mu=0.12347$ Pa.s and $\mu=0.0135$ Pa.s. These curves show that the decrease of recess depth leads concentrated pressure peaks around to the restrictor area and become more obvious at the very smaller recess depth. The same findings have been observed for two viscosities except that, in its smaller value, inertia effect is more accentuated. It must be noticed that the pressure in the recess area is not uniform when the deep recess decreases.

\section{Conclusion}

The performance of pressure field inside recess of hydrostatic bearing flat pad feeded by an orifice restrictor was analyzed using the 3DNavier-Stokes equations. The solution was obtained via a commercial code CFDAnsys CFX12.01, which uses a finite volume methodology to discretize the governing equations. In order to prove the robustness of the CFD resolution, a comparison with Reynolds equation was made at very deep recess. A very consistent superposition is found for both 

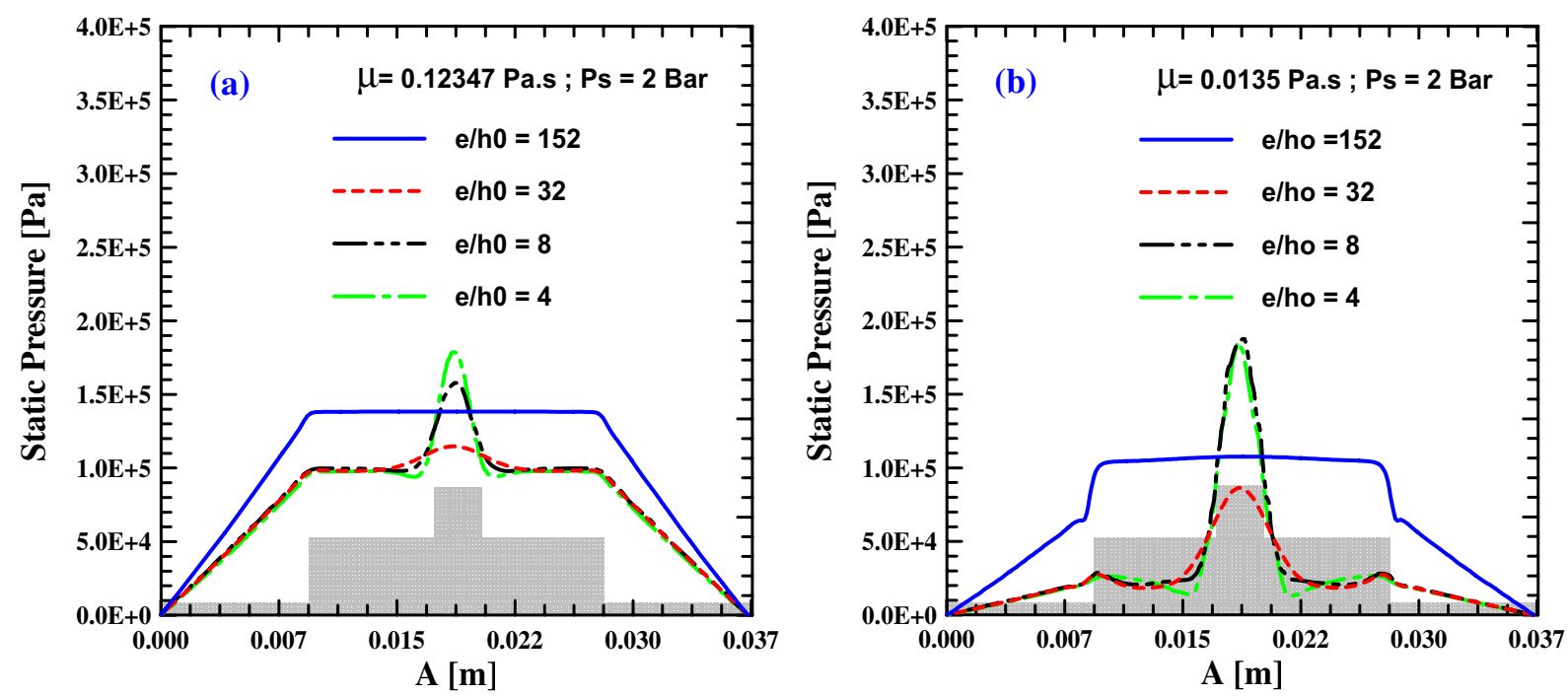

Fig. 9. Influence of the deep recess on static pressure predicted from CFD- ANSYS-CFX 12.0.

approaches. Then, a series of numerical studies were carried out to analyze the effect of pressure supply, dynamic viscosity and the recess depth on the pressure profile. Finally, analysis findings of this study can be summarized as follows:

- the increase of supply pressure decreases the film thickness and increases the Reynolds number and discharge coefficient. However, the film thickness and Reynolds number decrease with an increase in viscosity;

- an increase in viscosity and pressure ratio decreased significantly the Reynolds number and slightly the discharge coefficient;

- for the shallow recess $\left(e / h_{0}=4\right)$, the static pressure in the recess area is not uniform. It should be noticed that when the film thickness is considered as fixed, the increase of the supply pressure and the dynamic viscosity result in higher concentrated pressure peaks computed close to the restrictor area due to the high feeding Reynolds number. It should also be noticed that a pressure loss is observed between the restrictor area (injection zone) and the end of the recess that is due to the Rayleigh step effect results to the large developed Main Vortical Cell. These results were confirmed by Horvat and Frêne [21,22];

- for the deep recess $\left(e / h_{0}=152\right)$, the recess pressure distribution tends to become constant, the jet flow being completely diffused in the recess. It must be noticed that there are no concentrated pressure peaks around to restrictor area and no Rayleigh step effect. Further, the inertia effect already exists at the end of recess area when the dynamic viscosity had a lower value. It should also be noticed that when the film thickness is considered fix, the static pressure and recess pressure increase with the dynamic viscosity because;
- the decrease of recess depth leads concentrated pressure peaks around to the restrictor area and become more obvious at the very smaller recess depth.

\section{References}

[1] Y.S. Ho, N.S. Chen, Pressure distribution in a six-pocket hydrostatic journal bearing, Wear 98 (1984) 89-100

[2] J.K. Scharrer, K. Joseph, J.R. Hibbs, I. Robert, Flow coefficients for the orifice of a hydrostatic bearing, Tribol. Trans. 33 (1990) 543-550

[3] M.J. Braun, M. Dzodzo, Effects of hydrostatic pocket shape on the flow pattern and pressure distribution, Int. J. Rotating Machinery 1 (1995) 225-235

[4] M.J. Braun, F.K. Choy, Y.M. Zhou, The effects of a hydrostatic pocket aspect ratio, supply orifice position, and attack angle on steady-state flow patterns, pressure, and shear characteristics, J. Tribol. 115 (1993) 678-685

[5] M.J. Braun, Y.M. Zhou, F.K. Choy, Transient flow patterns and pressures characteristics in a hydrostatic pocket, J. Tribol. 116 (1994) 139-146

[6] A. Bouzidane, M. Thomas, Equivalent stiffness and damping investigation of a hydrostatic journal bearing, Tribol. Trans. 50 (2007) 257-267

[7] A. Bouzidane, M. Thomas, An electrorheological hydrostatic journal bearing for controlling rotor vibration, Comput. Struct. 86 (2008) 463-472

[8] A. Bouzidane, M. Thomas, Nonlinear dynamic analysis of a rigid rotor supported by a three-pad hydrostatic squeeze film dampers, Tribol. Trans. 56 (2013) 717-727

[9] A. Bouzidane, M. Thomas, Nonlinear dynamic behavior of a flexible shaft supported by smart hydrostatic squeeze film dampers, J. Tribol. 135 (2013) 031701

[10] M.J. Braun, M. Dzodzo, Effects of the feedline and the hydrostatic pocket depth on the flow pattern and pressure distribution, J. Tribol. 117 (1995) 224-232

[11] S.C. Sharma, R. Sinhasan, S.C. Jain, Performance of hydrostatic/hybrid journal bearings with unconventional recess geometries, Tribol. Trans. 41 (1998) 375-381 
[12] I. Pierre, M. Fillon, Influence of geometric parameters and operating conditions on the thermohydrodynamic behaviour of plain journal bearings, Proceedings of the Institution of Mechanical Engineers, Part J: J. Eng. Tribol. 214 (2000) 445-457

[13] C.H. Chen, Y. Kang, C.C. Huang, The influences of orifice restriction and journal eccentricity on the stability of the rigid rotor-hybrid bearing system, Tribol. Int. 37 (2004) 227-234

[14] S.K. Kakoty, B.C. Majumdar, Effect of fluid film inertia on stability of flexibly supported oil journal bearings: A non-linear transient analysis, Tribol. Trans. 45 (2002) 253-257

[15] D. Peloso, Analysis of fluid flow in a single pocket hydrostatic journal bearing, Mech. Eng. (2001) 200

[16] M.B. Dzodzo, M.J. Braun, Three dimensional flow and pressure patterns in a hydrostatic journal bearing, In: NASA Conference Publication, 1995
[17] M.J. Braun, M.B. Dzodzo, Three-dimensional flow and pressure patterns in a hydrostatic journal bearing pocket, J. Tribol. 119 (1997) 711-719

[18] W. Vieser, T. Esch, F. Menter, Heat transfer predictions using advanced two-equation turbulence models, CFX Validation Report, Report No. CFX-VAL 10 (2002) 0602

[19] Roberto Bassani, B. Piccigallo, Hydrostatic Lubrication, Tribology Series, 1992

[20] F.E. Horvat, M.J. Braun, Comparative experimental and numerical analysis of flow and pressure fields inside deep and shallow pockets for a hydrostatic bearing, Tribol. Trans. 54 (2011) 548-567

[21] J. Frêne, M. Arghir, V. Constantinescu, Combined thinfilm and Navier-Stokes analysis in high Reynolds number lubrication, Tribol. Int. 39 (2006) 734-747

[22] H.C. Rippel, Cast Bronze Hydrostatic Bearing Design Manual, Cast Bronze Bearing Institute, Inc., Cleveland, Ohio, 1964 